\title{
Uso da Cannabis sativa do tratamento da epilepsia - uma revisão da literatura
}

\author{
Use of Cannabis sativa in the treatment of epilepsy - a literature review \\ Uso de Cannabis sativa en el tratamiento de la epilepsia: revisión de la literatura
}

Recebido: 30/10/2021 | Revisado: 10/11/2021 | Aceito: 13/11/2021 | Publicado: 24/11/2021

\author{
Henrique Luan Vilela Lima \\ ORCID: https://orcid.org/0000-0003-0105-2171 \\ Centro Universitário Unifavip Wyden, Brasil \\ E-mail: henriquevilela19@gmail.com \\ Jeilsa da Silva Santos \\ ORCID: https://orcid.org/0000-0003-1646-4208 \\ Centro Universitário Unifavip Wyden, Brasil \\ E-mail: jeilsa2014@hormail.com \\ José Edson de Sousa Silva \\ ORCID: https://orcid.org/0000-0003-4926-4747 \\ Centro Universitário Unifavip Wyden, Brasil \\ E-mail: joseedson23@hotmail.com
}

\begin{abstract}
Resumo
A epilepsia é um distúrbio neurológico crônico, onde acontece descargas elétricas anormais, causando convulsões nos acometidos com essa patologia. Há alguns séculos se sabe do poder medicinal que a Cannabis sativa possui, mas nos últimos anos pode-se perceber o aumento de estudos da utilização de um de seus compostos, o canabidiol, para o tratamento de várias doenças, como Parkinson, Alzheimer, Esclerose Múltipla, ansiedade, depressão e principalmente na epilepsia. Este trabalho é uma revisão da literatura com o propósito de reunir informações e descrevê-las de forma clara e objetiva, fornecendo uma visão mais ampla sobre a utilização da Cannabis sativa no tratamento da epilepsia. Os dados foram coletados através de documentos, livros, artigos, revistas e textos, material disposto nos bancos de dados. Obtendo como conclusão, que o canabidiol possui capacidade terapêutica para o tratamento de pacientes com epilepsia e crises convulsivas, porém ainda precisa-se de novos estudos para compreender os compostos da Cannabis sativa, tanto para o tratamento, quanto dos seus efeitos colaterais nessa doença.
\end{abstract}

Palavras-chave: Cannabis; Epilepsia; Canabidiol; Crises convulsivas.

\begin{abstract}
Epilepsy is a chronic neurological disorder, where abnormal electrical discharges occur, causing seizures in those affected with this pathology. The medicinal power of Cannabis sativa has been known for several centuries, but in recent years there has been an increase in studies on the use of one of its compounds, cannabidiol, for the treatment of various diseases, such as Parkinson's, Alzheimer's, Sclerosis Multiple, anxiety, depression and especially in epilepsy. This work is a literature review with the purpose of gathering information and describing it in a clear and objective way, providing a broader view on the use of Cannabis sativa in the treatment of epilepsy. Data were collected through documents, books, articles, magazines and texts, material available in the databases. The conclusion is that cannabidiol has therapeutic capacity for the treatment of patients with epilepsy and convulsive crisis, but further studies are still needed to understand the compounds of Cannabis sativa, both for the treatment and its side effects in this disease.
\end{abstract}

Keywords: Cannabis; Epilepsy; Cannabidiol; Convulsive crises.

\section{Resumen}

La epilepsia es un trastorno neurológico crónico, en el que se producen descargas eléctricas anormales que provocan convulsiones en los afectados por esta patología. El poder medicinal de la Cannabis sativa se conoce desde hace varios siglos, pero en los últimos años se ha incrementado los estudios sobre el uso de uno de sus compuestos, el cannabidiol, para el tratamiento de diversas enfermedades, como Parkinson, Alzheimer, Esclerosis Múltiple, ansiedad, depresión y especialmente en epilepsia. Este trabajo es una revisión de la literatura con el propósito de recopilar información y describirla de forma clara y objetiva, proporcionando una visión más amplia sobre el uso de Cannabis sativa en el tratamiento de la epilepsia. Los datos fueron recolectados a través de documentos, libros, artículos, revistas y textos, material disponible en las bases de datos. La conclusión es que el cannabidiol tiene capacidad terapéutica para el tratamiento de pacientes con epilepsia y convulsiones, pero aún se necesitan más estudios para comprender los compuestos de Cannabis sativa, tanto para el tratamiento como sus efectos secundarios en esta enfermedad.

Palabras clave: Canabis; Epilepsia; Cannabidiol; Crisis convulsivas. 


\section{Introdução}

A epilepsia é um distúrbio de hiperexcitabilidade crônica, onde tem origem em diversas deficiências nas redes neuronais do cérebro, levando a convulsões frequentes. As crises são descargas elétricas irregulares que podem derivar de uma variedade de locais no cérebro e são capazes de promover desequilíbrios no comportamento, sensações e consciência. Em decorrência da super excitação e hiper sincronia de alguma rede neural, surgem as crises espontâneas que normalmente envolvem estruturas corticais e subcorticais, aliás crises epilépticas podem ser denominadas como generalizadas ou parciais (Reddy \& Golub, 2016).

Ao longo da convulsão, acontecem diversas contrações musculares, que duram em média de 5 a 7 minutos. Avalia-se que a prevalência mundial de epilepsia ativa esteja cerca de $0,5 \%$ a 1,0\% da população, e que por volta de 30\% dos indivíduos mesmo fazendo o uso do tratamento convencional, continua a apresentar episódios de crises. (Betting \& Guerreiro, 2008; Kwan \& Brodie, 2000)

A epilepsia não tem cura, mas pode ser controlada com medicamentos anticonvulsivantes, como Topiramato e Fenitoína 70\% dos indivíduos conseguem equilibrar as crises, em contra partida 30\% dos pacientes com lesões focais precisam de outras formas para diminuir as crises, em consequência da falta da eficácia desejada com os anticonvulsivantes convencionais. (Kruse et al.,2015).

A planta Cannabis Sativa conhecida popularmente como maconha, tem sua origem na Asia Central com grande poder de adaptação em relação ao clima, solo e altitude. $\mathrm{O}$ seu arbusto mede cerca de 2,5 a 4 metros de altura, possui uma folhagem bem característica composta por palmípedes de bordas serrilhadas bem peculiares e que exala um odor similar a fragrância Pachouli.A. O cultivo dessa erva é bastante simples requer água, pouco fertilizante, pouca terra e bastante luz solar ou artificial e sua germinação ocorre geralmente no verão ou na primavera (Moura et al., 2017).

A espécie faz parte da família da Cannabaceae e pode ser de três tipos: Cannabis sativa, Cannabis ruderallis e Cannabis indica. Devido à hibridização se torna um pouco difícil a distinção entre elas, no entanto o ciclo da C. ruderallis é consideravelmente curto, produz pouco THC ( $\Delta$ 9-tetraidrocanabinol), porém possui grande quantidade de CBD (canabidiol) (Amar, 2006).

A cannabis é utilizada de forma medicinal há milhares de anos, existem registros que seu uso mais antigo foi na china desde 2700 a.C. Na época as formas de aplicação incluíam: epilepsia, distúrbios no aparelho reprodutor feminino, alívio de sintomas mentais, malária, alívio de constipação, expectoração e tuberculose (Grotenhermen \& Russo, 2002).

Já na Índia a aplicabilidade farmacológica e religiosa dessa planta teve seu início por volta de 1000 anos a.C. A maconha era usada para várias funções, como anticonvulsivante (epilepsia), anestésico, anti-inflamatório, analgésico (nevralgia, dor de cabeça, dor de dente) e afrodisíaco (Zuard, 2006).

Após esse período, na Grã- Bretanha a eficácia da Cannabis se tornou mais conhecida em decorrência das suas propriedades terapêuticas, um médico chamado O'Shaughnessy que atuava na Índia, analisou o emprego da planta para o tratamento de epilepsia, raiva e reumatismo. (Atance \& Ruiz, 2000).

De volta a Inglaterra, ele trouxe consigo a tintura de cannabis que eram extratos de resina canábica dissolvidos em etanol para pacientes com tétano, convulsões e raiva. Em alguns testes foram administradas altas dosagens dessa tintura e constatou-se que ela foi eficaz no controle de espasmos em pessoas com tétano e raiva, inclusive apresentou resultados satisfatórios em casos de convulsões em crianças (Atance \& Ruiz, 2000).

Entretanto o princípio ativo dessa erva não era muito conhecido até encontrarem a presença dos canabinóides, e a descoberta dos seus ligantes endógenos em terminações nervosas, após esse episódio começou rapidamente a busca pela compreensão da função do sistema endocanabinóides e a sua conexão com patologias no organismo humano (Kupczyk, et al., 2009). 
Tendo isso em consideração, levantou-se novas pesquisas que indicam o impacto dos benefícios da utilização dos canabinóides em especial o Canabidiol, para pessoas com epilepsia, onde a farmacoterapia clássica não supre os efeitos esperados (Kruse et al.,2015).

Para prevenir outras convulsões de longa duração, os pais devem ser encorajados a administrar benzodiazepina oral / nasal / retal - diazepam ou midazolam para qualquer convulsão com duração superior a alguns minutos. No entanto, a repetição das convulsões permite que o diagnóstico seja considerado, e não mais as convulsões febris complicadas, mas a epilepsia provavelmente relacionada a uma mutação em SCN1A. O diagnóstico geralmente se torna possível antes do final do primeiro ano de vida e levanta a questão do tratamento mais adequado. (Chiron \& Dulac, 2011, p. 72).

Nos últimos anos, foi administrada uma série de medicamentos: fenobarbital, valproato, fenitoína e benzodiazepínicos, como clonazepam ou nitrazepam é mais do que clobazam para convulsões; etossuximida, piracetam e benzodiazepínicos usados para mioclonia; clorazepato, metsuximida, acetazolamida, alopurinol, sulthiame e zonisamida para outros tipos de crises epilépticas, a vigabatrina é ocasionalmente usada na puberdade. No entanto, os benefícios de todos esses compostos são moderados. (Dravet \& Bureau, 2008, citado em Chiron \& Dulac, 2011)

Como já foi apontada a existência do sistema endocanabinóides no cérebro de mamíferos, concluiu-se que ele é um complexo com dois receptores interligados a proteína G, que é o CB1 e CB2. (Saito, et al., 2010).

Formando assim um grupo de mensageiros neurais que são produzidos sob demanda e liberados por neurônios póssinápticos para inibir a liberação de neurotransmissores clássicos de terminais pré-sinápticos. Essa regulação controla uma variedade de funções neuronais como a ansiedade, medo e humor (Saito, et al., 2010).

Nesses últimos tempos, o Canabidiol puro tem ganhado uma atenção especial da comunidade médica e da mídia. Por apresentar um potencial promissor de tratamento para epilepsia pediátrica intratável, estudos com crianças que não demonstram resultados efetivos em terapias com drogas antiepiléticas, que fizeram o uso do CBD exibiram uma inibição significativa da ocorrência de crises ou a cessação total das mesmas, tendo uma melhora considerável da qualidade de vida (Crippa et al., 2016; TZADOK et al., 2016).

\section{Metodologia}

O presente estudo foi baseado em uma pesquisa bibliográfica do tipo revisão de literatura integrativa sobre a atuação da Cannabis sativa e como ela pode colaborar no tratamento da epilepsia. O estudo foi realizado no Centro Universitário do Vale do Ipojuca - UNIFAVIP/WYDEN, localizado na Av. Adjar da Silva Casé, no 800 - Indianópolis 55.024-740 Caruaru PE.

A revisão integrativa é uma abordagem que permite uma inclusão de diversas metodologias, seja ela experimental ou não experimental, desempenhar um papel maior na prática baseada em evidências para a saúde. a revisão integrativa contribui para a exibição de possibilidades variadas sobre um fenômeno preocupante e que tem tido como importante para a ciência e área da saúde (Whittemore \& Knafl, 2005).

Os dados foram coletados através de documentos, artigos, revistas e textos material disposto nos bancos de dados SciElo, Pubmed, Lilacs, Microsoft Academic e Google Acadêmico. Foram incluídos os documentos, revistas, artigos e outros com as seguintes descrições: Canabinóides na psiquiatria, história da Cannabis, tratamento da epilepsia com canabidiol. E estarão excluídos todos que estiverem fora desse grupo.

O período de artigos, documentos, revistas e outros que foram pesquisados para a fundamentação dessa revisão de literatura estará entre o ano 2000 a 2020. A análise e interpretação dos resultados foi do tipo integrativa com o propósito de apresentar resultados obtidos em pesquisas sobre um tema, de maneira abrangente. 
Foi uma revisão da literatura com o propósito de reunir informações e descrevê-las de forma clara e objetiva, fornecendo uma visão mais ampla sobre a utilização da Cannabis sativa no tratamento da epilepsia. Os estudos empregados nesse projeto são devidamente referenciados de acordo com as especificações da Associação Brasileira de Normas Técnicas (ABNT), respeitando a norma brasileira regulamentadora (NBR) 6023. Cumprindo com os direitos autorais e de propriedade intelectual do material pesquisado.

\section{Resultados e Discussão}

De acordo com Conselho Federal de Medicina (2014), nos últimos 40 anos, vem sendo realizadas pesquisas experimentais que demostraram que o canabidiol alcança um grande número de ações farmacológicas e dentre elas, está a epilepsia.

Segundo um estudo realizado pela Universidade de Stanford, em que foi realizada uma pesquisa onde um questionário de 24 perguntas foi apresentado a 150 pais, que apoiam o uso de cannabis enriquecida com canabidiol para tratar convulsões em seus filhos com epilepsia resistente ao tratamento, após quatro semanas, dezenove respostas perfizeram os critérios de inclusão, onde os dados do estudo foram coletados e hospedados na Research Electronic Data Capture (REDCap) e Stanford Center for Clinical Informatics, respectivamente.

A duração das administrações de cannabis enriquecida com canabidiol foi de mais ou menos um ano. Dezesseis pais relataram uma redução da frequência das convulsões de seus filhos, dois revelaram que seus filhos ficaram livre de convulsões após de pouco mais de quatro meses com o uso dessa cannabis, oito pais disseram que houve uma redução maior que $80 \%$ na frequência das crises, três relataram que obtiveram uma redução superior a $50 \%$ na frequência das crises, três disseram que houve uma redução superior a $25 \%$ na frequência dessas crises e três pais relataram nenhuma mudança na frequência, conforme o Gráfico 1. Doze pais conseguiram fazer o desmame de seus filhos em relação ao uso de drogas antiepiléticas.

Outros efeitos benéficos também foram apresentados pelos pais depois do uso dessa cannabis enriquecida, 79\% relataram melhor humor, $74 \%$ aumento da atenção, $68 \%$ um melhor sono e 32\% diminuição da autoestimulação. Quanto aos efeitos colaterais, somente $37 \%$ falaram de sonolência e $16 \%$ de fadiga.

Em outro estudo, foi realizado um ensaio com vários centros, randomizado, duplo-cego, e comparado com placebo. Esse ensaio envolveu 43 centros clínicos, em que somente 38 centros inscreveram pacientes entre 2 a 18 anos com síndrome de Dravet (conhecida também como epilepsia mioclônica grave da infância), com convulsões não totalmente controladas por drogas antiepiléticas, e que tivessem no mínimo 4 crises convulsivas durante um período de 14 semanas.

Após uma triagem, os pacientes que preencheram os critérios definidos, foram randomizados e obtiveram $100 \mathrm{mg} / \mathrm{mL}$ de canabidiol purificado ou solução placebo. Essas medicações foram administradas duas vezes ao dia em 2 doses divididas, iniciando com $2.5 \mathrm{mg} / \mathrm{kg} / \mathrm{d}$, chegando até a $10 \mathrm{mg} / \mathrm{kg} / \mathrm{dia}$ e $20 \mathrm{mg} / \mathrm{kg} / \mathrm{dia}$, podendo ser ajustadas as doses. Houve visitas clínicas nas semanas 2, 4, 8 e 14 depois da randomização e chamadas telefônicas nas semanas 6 e 10.

Foram elegidos 198 pacientes, onde 104 eram mulheres e 94 eram homens, no qual 65 receberam placebo, 66 receberam a dose de $10 \mathrm{mg} / \mathrm{kg} / \mathrm{dia}$ (chamado de CBD10) e 67 receberam a de $20 \mathrm{mg} / \mathrm{kg} / \mathrm{dia}$ (denominado CBD20). Nove pacientes foram retirados por descontinuação do estudo. Depois de um 14 semanas, foi verificado que houve uma redução percentual da linha de base na frequência das crises convulsivas, de 26,9\% para o grupo placebo, de 48,7\% para o grupo CBD10 e 45,7\% para o grupo CBD20, conforme o Gráfico 2. Também foi verificado que se obteve uma redução de 50\% de crises convulsivas em 29 pessoas do grupo CBD10, 33 para o CBD20 e de 17 do grupo placebo; e na redução de $75 \%$ das crises foi de 20 pessoas para o CBD10, 12 para o CBD20 e 4 para o grupo placebo.

Quanto aos efeitos adversos, 98 pacientes relataram efeitos considerados leves, 64 efeitos moderados e apenas 14 com efeitos graves. 
Outro estudo elaborado pelo Dr. Orrin Devinsky, neurologista especializado no tratamento de epilepsia e diretor do Comprehensive Epilepsy Center; na New York University School of Medicine, autorizado pela Federal Drug Administration (FDA), dirigiu um estudo aberto com o Epidiolex, cujo fármaco tem $98 \%$ de CBD. Essa pesquisa foi realizada com 23 pacientes, com idade de 10 anos e com uma dose diária sendo aos poucos aumentada até $25 \mathrm{mg} / \mathrm{kg} / \mathrm{dia}$ e associada com os medicamentos antiepiléticos já utilizados pelos pacientes.

O resultado foi que os $39 \%$ dos pacientes obtiveram uma redução de cerca de $50 \%$ das crises epiléticas, 3 de 9 pacientes com síndrome de Dravet e 1 de 14 pacientes com outra forma de epilepsia tiveram um controle total das crises epiléticas. Em relação aos efeitos colaterais recorrentes foram, fadiga, sonolência, diarreia, perca ou ganho do peso e redução ou aumento do apetite.

Gráfico 1. Relação dos Resultados Obtidos do estudo realizado pela Universidade de Stanford.

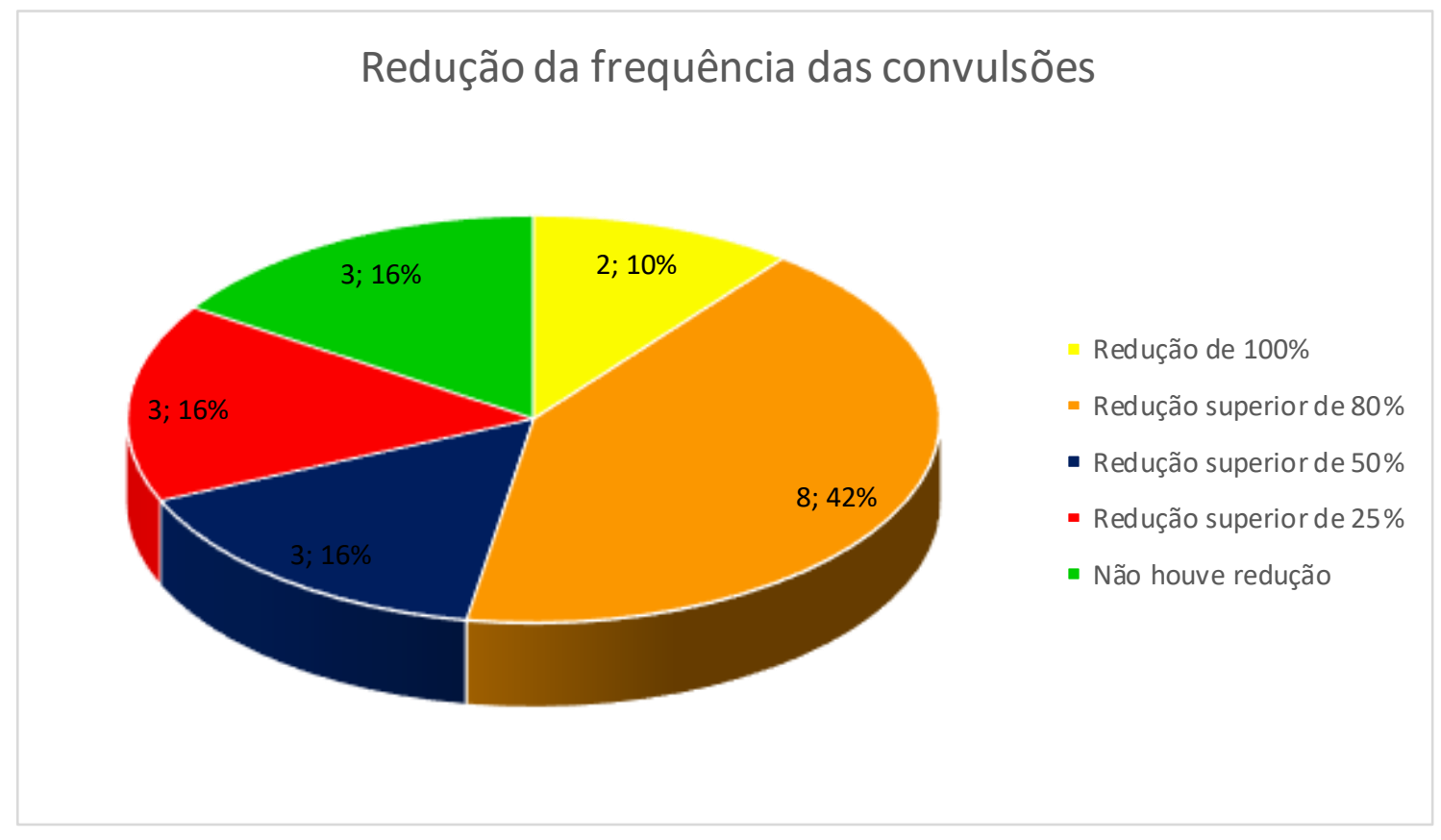

Fonte: Elaborado pelos autores (2021). 
Gráfico 2. Relação em porcento da redução da frequência de crises convulsivas de pacientes com a síndrome de Dravet.

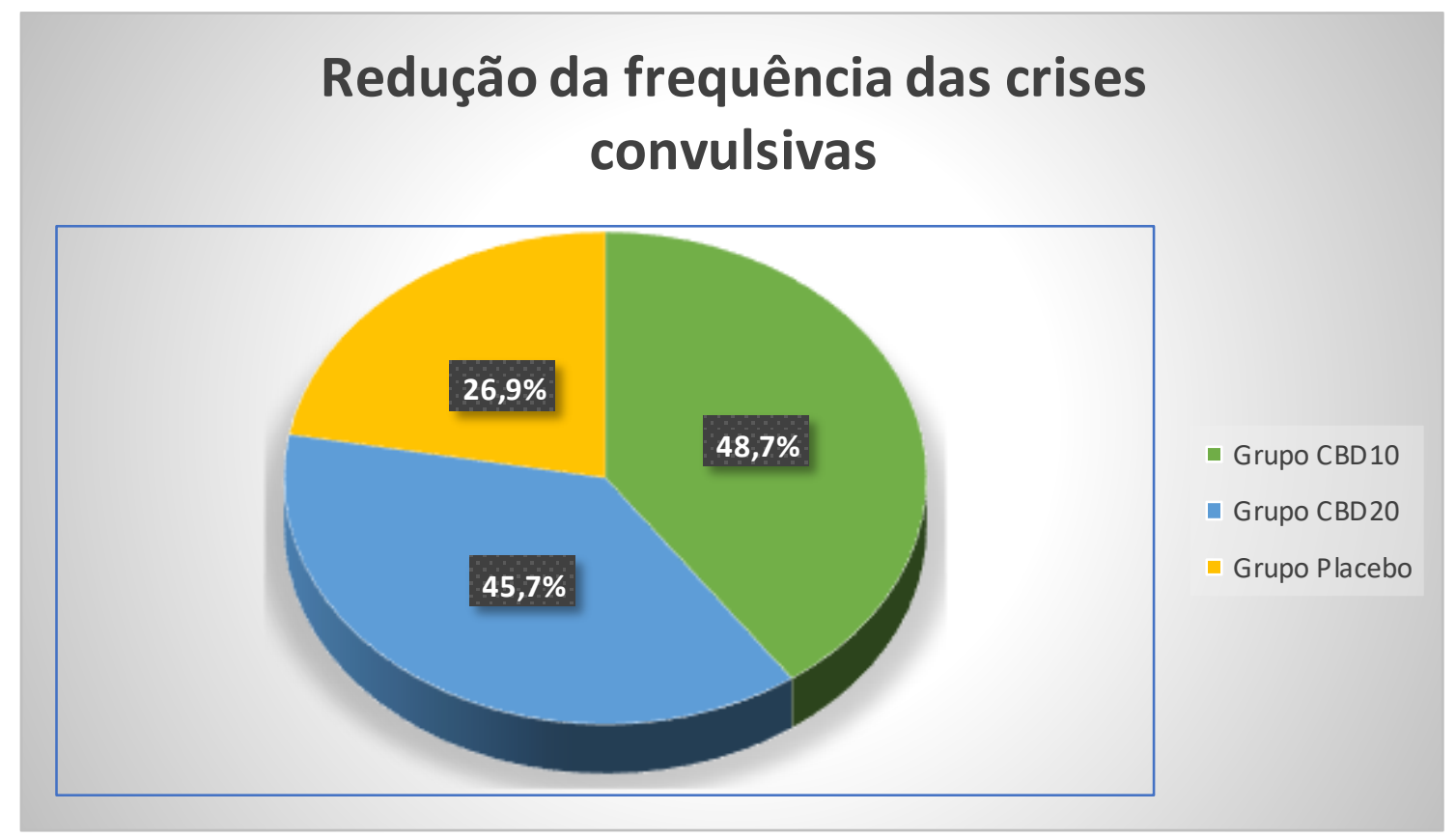

Fonte: Elaborado pelos autores (2021).

Conforme visto nos estudos acima, pode-se conferir que os estudos possuem um método parecido, mas não iguais, visto que o primeiro estudo apresentado é realizado com pacientes resistentes ao tratamento convencional, no segundo, o estudo é efetuado somente com pacientes com síndrome de Dravet, já no terceiro é elaborado não diz todas as formas de epilepsia; enquanto o primeiro e segundo estudo foi elaborado fazendo uma comparação com o placebo, já o terceiro não fornece dados suficientes para alegar que o estudo foi realizado com comparação ao placebo eles. Porém apesar dessa pequena diferença de métodos, pode-se inferir que os três estudos possuem uma semelhança nos resultados encontrados conferindo uma eficácia do CBD, seja o mesmo associado a outras drogas epiléticas ou somente ele.

\section{Conclusão}

Como sabe-se, a Cannabis sativa possui diversos outros compostos, e que um deles é o canabidiol que não apresentam efeitos psicoativos típicos da planta. De acordo com os dados levantados nesses estudos, pode-se confirmar que o canabidiol é um ativo que tem a capacidade terapêutica para o tratamento de pacientes com epilepsia e crises convulsivas.

Com base nesses dados apresentados, pesquisas validam a ideia que o canabidiol é um fármaco contemporâneo para o tratamento dessa patologia. Todavia, no Brasil esse composto ainda é tido como tabu, uma vez que a maconha até então é vista somente uma droga de abuso, seja ainda pela pouca disseminação de informações sobre ela ou pelos poucos estudos realizados nesse país, o que acaba limitando o acesso dos indivíduos ao tratamento.

Visto isso, é necessário destacar a necessidade e a relevância de novos estudos para uma melhor compreensão da Cannabis Sativa e de seus compostos quanto a epilepsia e seus efeitos colaterais, e proporcionar uma melhor qualidade de vida para pessoas que tem essa patologia.

\section{Agradecimentos}

Somos gratos à Deus, que encheu nossos corações de luz, contribuiu com a nossa cumplicidade e por me permitir ultrapassar todos os obstáculos encontrados ao longo dos nossos anos de estudo. Agradecemos aos familiares, por todo amor e 
carinho, e por entenderem os momentos de ausência. A todos os professores, por todos os conselhos, pela ajuda e pela paciência com a qual guiaram o nosso aprendizado, em especial José Edson de Sousa Silva, que foi o nosso orientador. Aos nossos colegas de sala, muito obrigado pela ajuda e pelas alegrias em vários momentos de dificuldade.

\section{Referências}

Atance, J. A. R., \& Ruiz, J. F. (2000). Uso de los cannabinoides a través de la historia. Adicciones, 12(5), 19-30. https://doi.org/10.20882/adicciones.670

Ben Amar, M. (2006). Canabinóides na medicina: uma revisão de seu potencial terapêutico. Journal of Ethnopharmacology, 105 (1-2), 125. https://doi.org/10.1016/j.jep.2006.02.001

Betting, L. E., \& Guerreiro, C. A. M. (2008). Tratamento das epilepsias generalizadas idiopáticas. Journal of Epilepsy and Clinical Neurophysiology, 14(suppl 2), 20-24. https://doi.org/10.1590/s1676-26492008000600004

Brucki, S. M. D., Frota, N. A., Schestatsky, P., Souza, A. H., Carvalho, V. N., Manreza, M. L. G., Mendes, M. F., Comini-Frota, E., Vasconcelos, C., Tumas, V., Ferraz, H. B., Barbosa, E., \& Jurno, M. E. (2015). Cannabinoids in neurology - Brazilian Academy of Neurology. Arquivos de Neuro-Psiquiatria, 73(4), 371-374. https://doi.org/10.1590/0004-282x20150041

CFM - Conselho Federal de Medicina. (2014). Resolução CFM 2113/2014 aprova o uso compassivo do canabidiol para o tratamento de epilepsias da criança e do adolescente refratárias aos tratamentos convencionais. https://portal.cfm.org.br/canabidiol/motivos.php

Chiron, C., \& Dulac, O. (2011). The pharmacologic treatment of Dravet syndrome. Epilepsia, 52 Suppl 2, 72-75. https://doi.org/10.1111/j.15281167.2011.03007.x

Crippa, J. A., Zuardi, A. W., \& Hallak, J. E. (2010). Uso terapêutico dos canabinoides em psiquiatria [Therapeutical use of the cannabinoids in psychiatry]. Revista brasileira de psiquiatria, 32 Suppl 1, S56-S66. https://doi.org/10.1590/S1516-44462010000500009

Dravet C, Bureau M. (2008) Severe Myoclonic Epilepsy in Infancy (Dravet syndrome). In Engel J, Pedley TA (Eds) Epilepsy, a comprehensive textbook. 2nd ed. Lippincott Williams Wilkins, Philadelphia, pp. 2337-2342.

Friedman, D., \& Devinsky, O. (2015). Canabinoides no tratamento da epilepsia (Cannabinoids in the treatment of epilepsy). The New England Journal of Medicine, 1048-1058. https://www.nejm.org/doi/10.1056/NEJMra1407304

Grotenhermen, F., \& Russo, E. (2008). Cannabis and cannabinoids: pharmacology, toxicology, and therapeutic potential. Routledge.

Kruse, M., Souza, P., \& Toma, W. (2015). A importância do princípio ativo canabidiol (CBD) presente na Cannabis sativa L. no tratamento da epilepsia [Sessão de pôsteres]. IV Simpósio de Ciências Farmacêuticas, São Camilo. http://www.saocamilo-sp.br/novo/eventos-noticias/simposio/15/SCF014_15.pdf

Kupczyk, P., Reich, A., \& Szepietowski, J. C. (2009). Cannabinoid system in the skin - a possible target for future therapies in dermatology. Experimental dermatology, 18(8), 669-679. https://doi.org/10.1111/j.1600-0625.2009.00923.x

Kwan, P., \& Brodie, M. J. (2000). Early identification of refractory epilepsy. The New England journal of medicine, 342(5), 314-319. https://doi.org/10.1056/NEJM200002033420503

Miller, I., Scheffer, I. E., Gunning, B., Sanchez-Carpintero, R., Gil-Nagel, A., Perry, M. S., Saneto, R. P., Checketts, D., Dunayevich, E., Knappertz, V., \& GWPCARE2 Study Group (2020). Dose-Ranging Effect of Adjunctive Oral Cannabidiol vs Placebo on Convulsive Seizure Frequency in Dravet Syndrome: A Randomized Clinical Trial, JAMA neurology, 77(5), 613-621. https://doi.org/10.1001/jamaneurol.2020.0073

Moura, E. R.., Veras, L. B. S., Alencar, E. V. M., Frota, R. D. D. D., Santos, M. Y. B., \& Maceno, G. A. (2017). POTÊNCIAL TRAPÊUTICO DA CANNABIS SATIVA: CANABIDIOL E TETRAHIDROCANABIDIOL [Sessão de pôsteres]. Revista Interdisciplinar de Ciências Médicas - Anais, Teresina. http://docplayer.com.br/81118404-Potencial-trapeutico-dacannabis-sativa-canabidiol-etetrahidrocanabidiol.html

Porter, B. E., \& Jacobson, C. (2013). Report of a parent survey of cannabidiol-enriched cannabis use in pediatric treatment-resistant epilepsy. Epilepsy \& behavior: $E \& B, 29(3), 574-577$. https://doi.org/10.1016/j.yebeh.2013.08.037

Reddy, D. S., \& Golub, V. M. (2016). The Pharmacological Basis of Cannabis Therapy for Epilepsy. Journal of Pharmacology and Experimental Therapeutics, 357(1), 45-55. https://doi.org/10.1124/jpet.115.230151

Saito, V. M., Wotjak, C. T., \& Moreira, F. A. (2010). Exploração farmacológica do sistema endocanabinoide: novas perspectivas para o tratamento de transtornos de ansiedade e depressão? Brazilian Journal of Psychiatry, 32 Suppl 1, S7-S14. SciElo. https://www.scielo.br/j/rbp/a/dPP9G5tCc8NNkbBj6cbjcwk/?lang=pt

Tzadok, M., Uliel-Siboni, S., Linder, I., Kramer, U., Epstein, O., Menascu, S., Nissenkorn, A., Yosef, O. B., Hyman, E., Granot, D., Dor, M., Lerman-Sagie, T., \& Ben-Zeev, B. (2016). CBD-enriched medical cannabis for intractable pediatric epilepsy: The current Israeli experience. Seizure, 35, 41-44. https://doi.org/10.1016/j.seizure.2016.01.004

Whittemore, R., \& Knafl, K. (2005). The integrative review: updated methodology. Journal of advanced nursing, 52(5), 546-553. https://doi.org/10.1111/j.1365-2648.2005.03621.x

Zuardi, A. W. (2006). História da cannabis como medicamento: uma revisão. Revista Brasileira de Psiquiatria, 153-157 SciElo. https://www.scielo.br/j/rbp/a/ZcwCkpVxkDVRdybmBGGd5NN/abstract/?lang=en 